> La maladie de Pompe est une maladie lysosomale avec atteinte musculaire prédominante. On distingue la forme sévère du nourrisson avec troubles cardiaques des formes tardives de l'enfant, de l'adolescent et de l'adulte qui n'ont en général pas de manifestations cardiaques. L'association d'une maladie de Pompe et d'un «rigid spine » a été occasionnellement rapportée. Plusieurs profils ont été décrits. La présentation la plus fréquente est un déficit des muscles des ceintures et du diaphragme. Une scoliose est rapportée dans un tiers des patients, mais seulement $15 \%$ associent une raideur spinale. L'atteinte extra-musculaire, en particulier des symptômes cardio-cérébrovasculaires sont observés dans un tiers des patients. L’observation rapportée ici présente un tableau de myopathie avec un phénotype typique de raideur spinale sélective, associé à une augmentation modérée des enzymes musculaires, des signes myopathiques à la biopsie, et une atteinte cardiaque.<

\section{Observation}

Le patient est d'origine française et ses parents n'ont pas de lien de consanguinité connu. II a été suivi en neuropédiatrie dès l'âge de 10 ans pour crises convulsives et développé un tableau caractérisé par une faiblesse musculaire axiale avec décollement des omoplates, des rétractions articulaires, une scoliose dorsale et un rachis cervico-dorsal raide (rigid spine). Les enzymes musculaires étaient élévées (CPK 1200 $\mathrm{UI} / \mathrm{I}$ ). A 16 ans, une biopsie a montré des anomalies myopathiques non spécifiques. A 26 ans, on notait une fonction respiratoire altérée avec signes d'atteinte diaphragmatique (CV assis $70 \%$; CV couché $44 \%$ ). Son frère aîné présentait aussi des signes d'une myopathie rétractile et est décédé subitement vers l'âge de trente ans.

\title{
Orientation diagnostique d'un cas de «Rigid spine » familial par IRM musculaire corps entier
}

\author{
Eliana Cavassa ${ }^{1,2}$, Mickael Tordjman $^{1}$, Ana Ferreiro ${ }^{3}$, \\ Robert Carlier ${ }^{3,4}$, Susana Quijano-Roy ${ }^{5}$
}

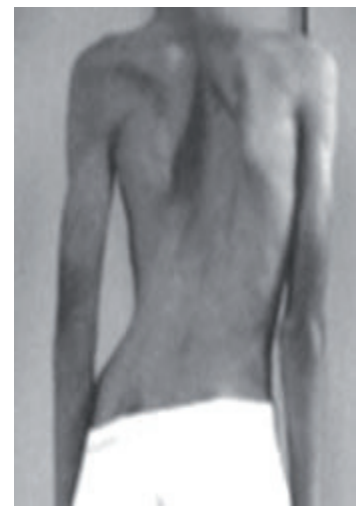

Les premiers signes d'atteinte musculaire chez le cas index étaient apparus à 11 ans avec une scoliose très évolutive et une faiblesse musculaire discrète. Les CPK étaient discrètement augmentées (370-600 UI/I). L'EMG montrait un tracé myogène avec activité spontanée sur le quadriceps. La biopsie musculaire était en faveur d'une myopathie, sans orientation spécifique. À 13 ans, le patient a rapporté une dyspnée à l'effort. II est opéré de sa scoliose à 15 ans (arthrodèse rachidienne). Au niveau moteur, l'évolution a été lente. À 30 ans il avait des difficultés pour la montée des escaliers et des difficultés progressives pour porter les bras au-dessus de la tête. En revanche, il a développé une insuffisance respiratoire restrictive sévère avec des signes de défaillance diaphragmatique qui a nécessité une ventilation nocturne non invasive à 31 ans. Au niveau cardiaque, il a eu un pacemaker à 36 ans en raison d'un bloc auriculo-ventriculaire de haut degré. A cet âge, il a un poids de $37 \mathrm{~kg}$. II marche avec le cou en hyperextension et le tronc en antépulsion. Le déficit musculaire prédomine au niveau axial. En particulier, les muscles fléchisseurs du cou sont très déficitaires et il a une hyperlordose cervicale raide. II se relève sans appui. Les muscles sternocléido-mastoïdiens et pectoraux sont très amyotrophiques et la cage 


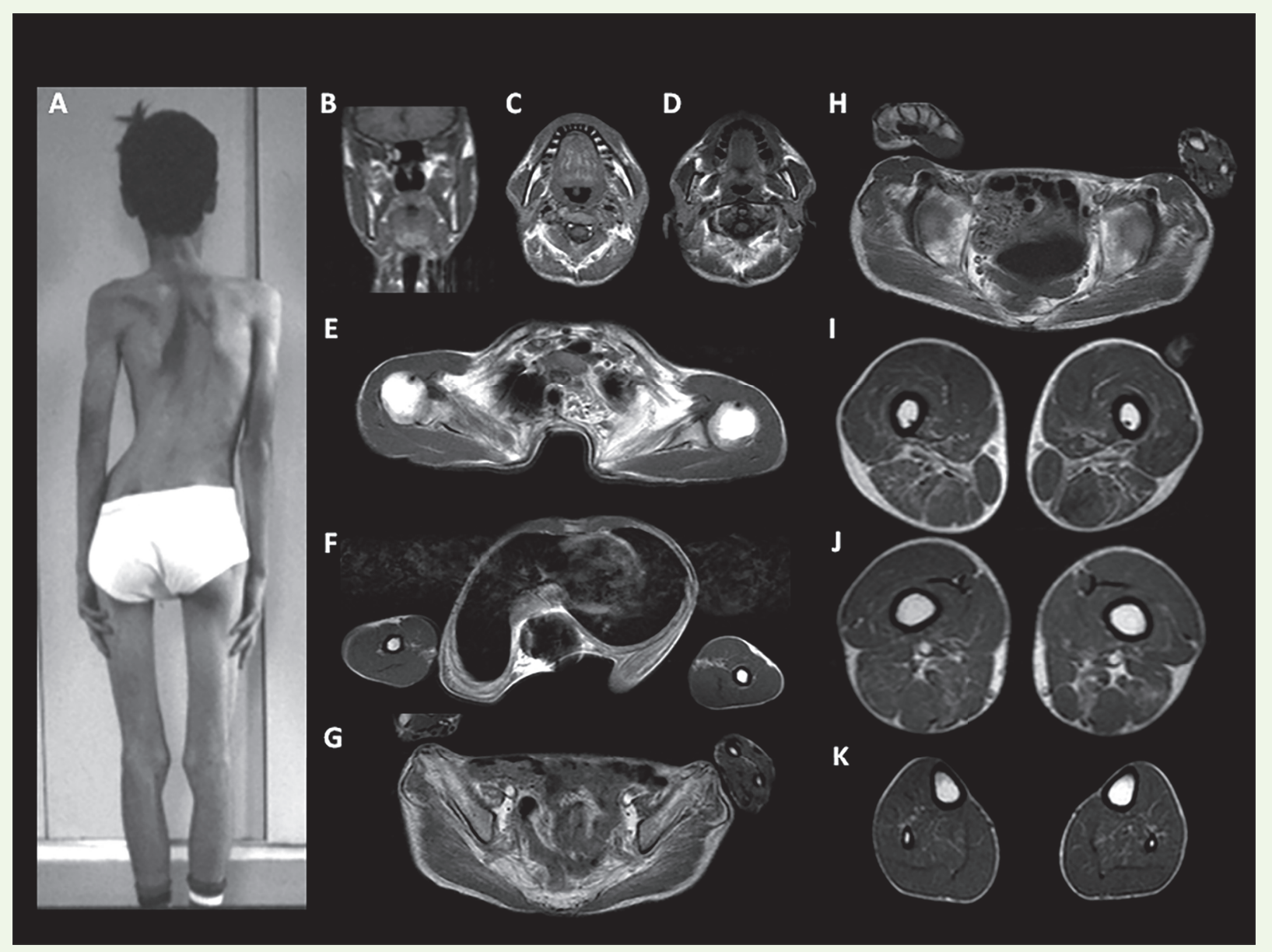

Figure 1. Patient avec maladie de Pompe : Aspect Clinique à 16 ans (A) et IRM corps entier à 35 ans (B-K). A : Aspect clinique caractérisé par une colonne raide, amyotrophie diffuse, dos creux. $B-K$ ) : IRM en séquence Tl- TSE à 35 ans: TÊTE : coupes frontale (B) et axiales au niveau de la tête $(C, D)$ qui montrent la conservation des muscles masticateurs mais une atteinte de la langue (flèches blanches longues dans $B, C$ ). Coupes axiales au niveau de la ceinture scapulaire qui montre une atteinte sélective du muscle sous-scapulaire (flèches noires dans $\varepsilon$ ) et pectoraux, avec une très bonne conservation des muscles deltoïdes et sus-épineux. TRONC : coupes axiales avec matériel d'arthrodèse qui empêchent de visualiser les muscles paravertébraux. MEMBRES SUPÉRIEURS, respect des muscles des bras, avant bras et mains ( $F, G, H)$ PELVIS : respect relative des muscles fessiers, avec atrophie relative des grands fessiers et discrète infiltration des muscles moyens et petits fessiers (G, $H$ ). MEMBRES INFÉRIEURS : Infiltration des grands adducteurs, semi-membraneux et biceps fémoral au niveau de la cuisse (J) et respect distal au niveau de la jambe $(K)$.

thoracique a un aspect étroit. À l'inverse, la force et la masse musculaire des membres inférieurs et supérieurs sont relativement bien conservées. Au niveau de la ceinture pelvienne, il présente un déficit marqué de la flexion des hanches et des fessiers. II a des rétractions articulaires au niveau des muscles ischio-jambiers et des poignets, sans hyperlaxité distale associée. La recherche de mutations des gènes LMNA, DES, FHLI, RYRI, SEPNI et ACTAl s'avère négative. Une nouvelle biopsie montre des signes myopathiques sans spécificité (Figure 1). Le diagnostic est finalement suggéré par les résultats de I'IRM musculaire de corps entier. Les anomalies des membres inférieurs ne sont pas très contributives ni spécifiques, mais l'atteinte sélective de la langue et des muscles sous-scapulaires et pectoraux sont typiques de la maladie de Pompe [1] et permettent d'orienter le diagnostic (deux mutations du gène GAA chez le patient et son frère ont été identifiées (c.-32-13T>G; c.655G>A, p.Gly219Arg).

\section{Commentaire}

La maladie de Pompe (aussi appelée glycogénose de type 2, déficit en alpha-glucosidase acide ou déficit en maltase acide), est une maladie lysosomale de surcharge génétique héréditaire avec une atteinte musculaire prédominante. On distingue la forme infantile 
sévère du nourrisson (avant l'âge de un an), qui s'accompagne d'une cardiomyopathie sévère, et des formes tardives de l'enfant ou de l'adolescent (forme tardive juvénile) et de l'adulte (forme tardive adulte) qui n'ont généralement que peu ou pas de manifestations cardiaques. La forme à révélation tardive, notamment chez l'adulte, est sous-estimée. La prévalence est très variable d'un pays à l'autre (1/138000 naissances pour la forme infantile; 1/ 57000 pour les formes tardives).

L'association d'un «rigid spine » et d'une maladie de Pompe a été occasionnellement rapportée [2-4]. Néanmoins, dans une série récente de 44 patients diagnostiqués avec une forme tardive, les auteurs décrivent plusieurs profils différents de manifestations musculo-squelettiques et cérébro-vasculaires [5]. La présentation la plus fréquente est un déficit des muscles des ceintures et du diaphragme (80\%). Une scoliose est rapportée dans un tiers des patients, mais seulement $15 \%$ associent une raideur spinale et un bas index corporel (15\%). Il est intéressant à signaler que l'atteinte extra-musculaire, en particulier des symptômes cardio-cérébrovasculaires sont observés dans un tiers des patients.

Le cas décrit présente un tableau de myopathie avec un phénotype typique de «rigid spine» (raideur spinale sélective), associé à une augmentation modérée des enzymes musculaires et des signes myopathiques à la biopsie, et une atteinte cardiaque. Les antécédents familiaux suggèrent une transmission récessive. L'atteinte respiratoire avec atteinte sélective du diaphragme chez un patient ambulatoire avec un début dans l'enfance sont typiques d'une myopathie par mutation du gène SEPNI mais l'analyse génétique ne confirme pas cette hypothèse. L'antécédent de décès subit chez le frère et l'apparition d'une arythmie cardiaque chez le cas index, associée à une raideur spinale et une hyperextension cervico-thoracique font soupçonner une laminopathie, mais le gène LMNA est normal chez le patient et son frère. Les dystrophies musculaires congénitales et myopathies par mutation des gènes COL6A1, COL6A2 et COL6A3 (Ullrich-Bethlem) et LAMA2 peuvent avoir dans leurs spectres moins sévères des phénotypes similaires avec acquisition de la marche, raideur spinale prédominant sans des rétractions articulaires significatives. Enfin, I'IRM corps entier révèle des anomalies qui ne sont pas en faveur de ces hypothèses diagnostiques, mais de la maladie de Pompe [6].

Cette observation souligne le caractère très protéiforme des manifestations cliniques de la maladie de Pompe et montre la contribution positive de I'IRM musculaire corps entier dans l'arsenal diagnostique.

Diagnostic orientation of « Rigid spine » familial case with whole body muscle MRI

\section{LIENS D'INTÉRÊT}

Les auteurs déclarent n'avoir aucun lien d'intérêt concernant les données publiées dans cet article.

\section{RÉFÉRENCES}

1. Carlier Ry, Laforet P, Wary C, et al. Whole-body muscle MRI in 20 patients suffering from late onset Pompe disease: involvement patterns. Neuromuscul Disord 2011 ; 21 : 791-9.

2. Kostera-Pruszczyk A, Opuchlik A, Lugowska A, et al. Juvenile onset acid maltase deficiency presenting as a rigid spine syndrome. Neuromuscul Disord $2006 ; 16: 282-5$.

3. Laforêt P, Doppler V, Caillaud C, et al. Rigid spine syndrome revealing lateonset Pompe disease. Neuromuscul Disord $2010 ; 20: 128-30$.

4. Panosyan FB, Fitzpatrick MF, Bolton CF. Late onset Pompe disease mimicking rigid spine syndrome. Can J Neurol Sci $2014 ; 41: 286-9$.

5. Schüller A, Wenninger S, Strigl-Pill N, Schoser B. Toward deconstructing the phenotype of late-onset Pompe disease. Am J Med Genet C Semin Med Genet $2012 ; 160 C: 80-8$.

6. Quijano-Roy S, Avila-Smirnow D, Carlier RY, WB-MRI muscle study group. Whole body muscle MRI protocol: pattern recognition in early onset NM disorders. Neuromuscul Disord 2012 ; 22 (suppl 2) : S68-84.

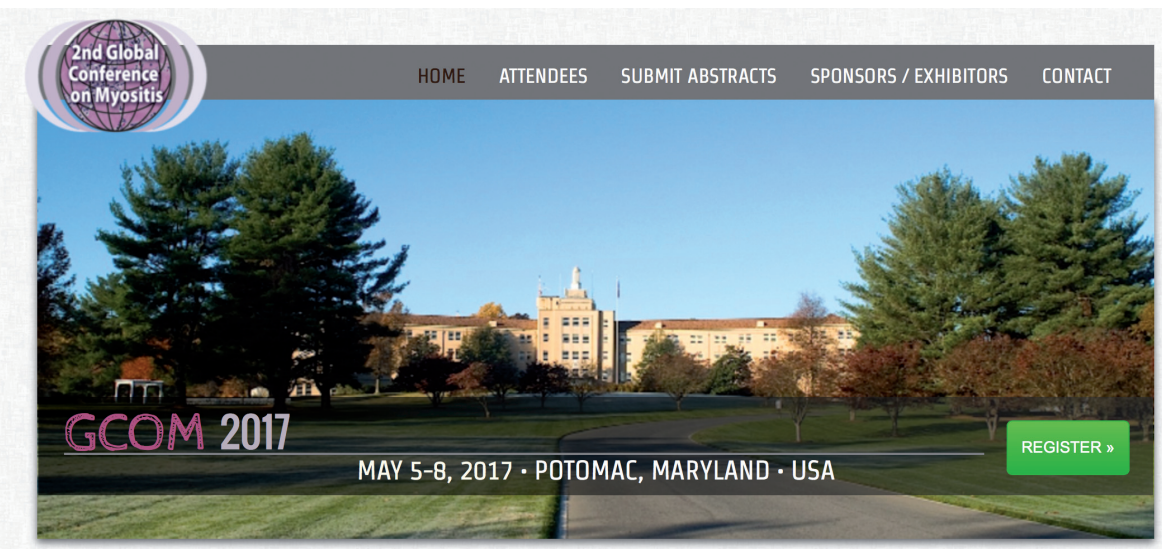

\section{2nd Global Conference on Myositis}

\section{TIRÉS À PART}

S. Quijano-Roy 